\title{
EFICIÊNCIA DE TRATAMENTOS SILVICULTURAIS POR ANELAMENTO NA FLORESTA DO JARI, AMAPÁ
}

\author{
Celso Paulo de Azevedo ${ }^{1}$, José Natalino Macedo Silva ${ }^{2}$, Cintia Rodrigues de Souza ${ }^{3}$, \\ Carlos Roberto Sanquetta ${ }^{4}$ \\ ${ }^{1}$ Eng. Florestal, Dr., Embrapa Amazônia Ocidental, Manaus, AM, Brasil - celso.azevedo@cpaa.embrapa.br \\ ${ }^{2}$ Eng. Florestal, Dr., Serviço Florestal Brasileiro, Brasília, DF, Brasil - natalino.silva @ florestal.gov.br \\ ${ }^{3}$ Eng $^{\underline{a}}$ Florestal, M.Sc., Embrapa Amazônia Ocidental, Manaus, AM, Brasil - cintia.souza@cpaa.embrapa.br \\ ${ }^{4}$ Eng. Florestal, Dr., Universidade Federal do Paraná, Curitiba, PR, Brasil - sanquetta@ufpr.br
}

Recebido para publicação: 31/05/2010 - Aceito para publicação: 28/11/2011

\begin{abstract}
Resumo
O anelamento é um tratamento silvicultural aplicado nas florestas tropicais com o objetivo de manter ou até aumentar a produtividade das espécies exploradas comercialmente para o próximo ciclo de corte. Neste trabalho, analisou-se o efeito de diferentes níveis de exploração e tratamentos silviculturais sobre a dinâmica da floresta remanescente na região do Jari/AP. O tratamento silvicultural foi realizado em 1994, aplicando-se dois tipos de desbaste: desbaste sistemático, com intensidades de redução da área basal original de 30\% e 50\% e o desbaste seletivo (anelamento com entalhes e aplicação de arboricida). Na aplicação do tratamento, foram aneladas em média 18,3 árvores.ha ${ }^{-1}, 2,1 \mathrm{~m}^{2} \cdot \mathrm{ha}^{-1}$ de área basal e $25,1 \mathrm{~m}^{3} \cdot \mathrm{ha}^{-1}$ de volume, com total de 495 indivíduos anelados (de 93 espécies). A quantidade de árvores mortas em consequência do anelamento variou consideravelmente entre os tratamentos. De maneira geral, o anelamento com a aplicação do arboricida mostrou maior efeito em função da espécie do que com o tamanho das árvores. Espécies com troncos sulcados apresentaram baixa mortalidade. Árvores com diâmetro inferior a $50 \mathrm{~cm}$ apresentaram mortalidade de 61 a $90 \%$. Nas classes diamétricas superiores, a taxa de mortalidade foi semelhante, em torno de 69 a $100 \%$.
\end{abstract}

Palavras-chave: Espécies nativas; tratamentos silviculturais; exploração florestal; Amazônia.

\begin{abstract}
Efficiency of silvicultural treatments by girdling in the Jari Forest, Amapá. The girdling is a silvicultural treatment applied in tropical forests in order to maintain or even increase the productivity of commercially exploited species to the next cutting cycle. In this research we analyzed effects of different levels of exploitation and silvicultural treatments on the dynamics of remaining forest in the Jari region/AP. The silvicultural treatment was improved in 1994 and applied to two types of thinning: systematic thinning, with intensities of original basal area reduction of $30 \%$ and $50 \%$ and selective thinning (with girdling slots and applying arboricida). In the application of treatment it were ringed on average 18.3 trees.ha ${ }^{-1}, 2,1 \mathrm{~m}^{2} \cdot \mathrm{ha}^{-1}$ of basal area and $25.1 \mathrm{~m}^{3} \cdot \mathrm{ha}^{-1}$ of volume, total of 495 treated individuals $(93$ species). The amount of dead trees as a result of girdling had important variation between treatments. In general, the girdling with application of arboricida presented greater effect in relation to the species than to trees sizes. Species with furrowed trunks presented low mortality. Trees with less than $50 \mathrm{~cm}$ of diameter had a mortality rate of 61 to 90 percent. In superior diameter classes the mortality rate was similar, around 69 to 100 percent.

Keywords: Native species; silvicultural treatments; forest exploration; Amazonia.
\end{abstract}

\section{INTRODUÇÃO}

A extração de madeira em florestas tropicais é uma atividade que causa grandes impactos ao ecossistema e, por isso, constitui o momento mais crítico na aplicação de um sistema de manejo visando à produção sustentável. O planejamento adequado dessa atividade é a chave para se obterem bons resultados, tanto do ponto de vista ambiental como econômico (SILVA et al., 2001).

A aplicação de técnicas que reduzem os impactos da atividade madeireira sobre a floresta 
residual constitui o primeiro passo para manter a produtividade das florestas tropicais. Entretanto, somente a aplicação dessas técnicas não é suficiente para manter a capacidade produtiva da floresta (SILVA, 2004).

$\mathrm{Na}$ busca por um sistema silvicultural adequado para as florestas tropicais, uma importante consideração é o baixo incremento das espécies de valor comercial madeireiro após a primeira exploração. Isso se dá devido à relativa escassez, em número e volume, de árvores potenciais para a próxima extração (árvores de espécies com valor comercial e diâmetro entre 30-45 cm ou mais) deixadas após a primeira exploração, aliada ao lento crescimento delas (DE GRAAF et al., 1999).

As taxas de crescimento podem ser aceleradas pelos tratamentos silviculturais, pois eles reduzem a competição por luz e nutrientes com as espécies mais abundantes e sem valor comercial. Esses tratamentos basicamente envolvem dois tipos: i) liberação ou desbaste seletivo, que consiste na remoção de indivíduos competidores, não desejáveis, cujas copas estejam competindo por luz com as copas das árvores de espécies selecionadas para a próxima colheita; ou ii) refinamento ou desbaste sistemático, que consiste na redução da área basal de espécies não desejáveis, visando diminuir a competição no povoamento, de forma geral.

Segundo Jardim et al. (1996), em termos silviculturais, a abertura do dossel pode ser feita de duas maneiras principais: através do abate de árvores, na exploração florestal ou nos desbastes, e através de técnicas de anelamento de árvores, com ou sem envenenamento.

A redução da vegetação sem aproveitamento comercial pode ser feita por meio da aplicação de uma técnica conhecida como anelamento, que consiste na retirada da casca e da entrecasca, formando um anel completo em torno do fuste da árvore, provocando uma descontinuidade nos elementos condutores. As árvores aneladas perdem, de forma lenta, primeiramente as folhas, depois os pequenos ramos e finalmente os galhos mais desenvolvidos (JARDIM et al., 1996). Consequentemente, quando a árvore está totalmente morta, sua queda diminui o impacto sobre a vegetação remanescente. Esse processo não implica brusca admissão de luz sobre o solo florestal, reduzindo ao mínimo as possibilidades de sua dessecação pela ação do sol e do vento.

Dubois (1971) descreve três modalidades de anelamento: anelamento simples (retirada da casca em anel completo), anelamento com entalhes (anelagem simples e entalhes feitos com machadinha ou terçado no anel) e anelamento profundo (retirada da casca e camada superficial do alburno com espessura em torno de $2 \mathrm{~mm}$ ). No entanto, a técnica de anelamento é pouco eficiente na eliminação de indivíduos de algumas espécies, principalmente aquelas com reentrâncias no fuste (COSTA et al., 2001). Para melhorar a eficiência do anelamento e acelerar a morte das árvores, pode-se aplicar arboricida no anel.

Segundo Oliveira et al. (2006), ainda há carência de conhecimentos sobre os efeitos em longo prazo das operações de manejo em florestas tropicais. Devido a problemas de ordem técnica, operacional ou financeira, somente um número reduzido de parcelas tem sido mantido e acompanhado por tempo suficiente para medir o impacto das operações de exploração sobre a vegetação remanescente.

Este trabalho teve como objetivo analisar a eficiência dos tratamentos silviculturais por meio de anelamento de árvores na Floresta da Jari, Amapá, buscando responder as seguintes questões: a) o tamanho das árvores tem alguma influência na eficiência dos tratamentos, e b) quais os efeitos dos tratamentos sobre as espécies.

\section{MATERIAL E MÉTODOS}

\section{Localização e caracterização da área}

Este estudo foi conduzido com dados do experimento originalmente denominado "Determinação da intensidade ideal de exploração para fins de manejo policíclico da floresta úmida densa", do Projeto Técnicas de Manejo para Florestas Tropicais Úmidas da Amazônia Visando Rendimento Sustentado, depois denominado Projeto Bom Manejo (Embrapa/CIFOR/ITTO) - manejo sustentável de florestas de produção em escala comercial na Amazônia brasileira, desenvolvido pela Embrapa Amazônia Oriental. O experimento foi iniciado em julho de 1983, em uma área de 500 ha de floresta densa na Companhia Florestal Monte Dourado (Jari), na localidade Morro do Felipe, município de Vitória do Jari, Estado do Amapá (Figura 1).

A Floresta da Jari está localizada entre as coordenadas $52^{\circ} 20^{\prime \prime} \mathrm{W}$ e $00^{\circ} 55^{\prime \prime} \mathrm{S}$, a uma altitude de aproximadamente $150 \mathrm{~m}$. O clima é do tipo Ami, pela classificação de Köppen. A precipitação média 
anual alcança $2.234 \mathrm{~mm}$, com período chuvoso de dezembro a maio. Ocorre uma estação seca de três meses, que se inicia em junho e que se caracteriza por uma precipitação mensal inferior a $8 \%$ do volume anual de chuva. A temperatura média anual é de $25,8{ }^{\circ} \mathrm{C}$, com a amplitude térmica variando mais ou menos $2{ }^{\circ} \mathrm{C}$ entre o máximo e o mínimo valor mensal. Na Jari, a vegetação é do tipo Floresta Ombrófila Densa. Os solos são do tipo Latossolo Amarelo Distrófico, com textura argilosa pesada.

\section{Histórico da área de estudo}

O experimento foi iniciado em 1983, com a realização do inventário pré-exploratório considerando todas as árvores com diâmetro à altura do peito (DAP) $\geq 50 \mathrm{~cm}$. Em 1985 foi realizada a exploração florestal na área de floresta primária, retirando-se $15 \%, 25 \%$ e $35 \%$ do volume total das árvores com DAP $\geq 50 \mathrm{~cm}$, correspondendo, aproximadamente, a $25 \mathrm{~m}^{3} / \mathrm{ha}, 40 \mathrm{~m}^{3} / \mathrm{ha}$ e $60 \mathrm{~m}^{3} / \mathrm{ha}$, respectivamente. Foram exploradas árvores com DAP $\geq 60 \mathrm{~cm}$, de 42 espécies de valor comercial na região.

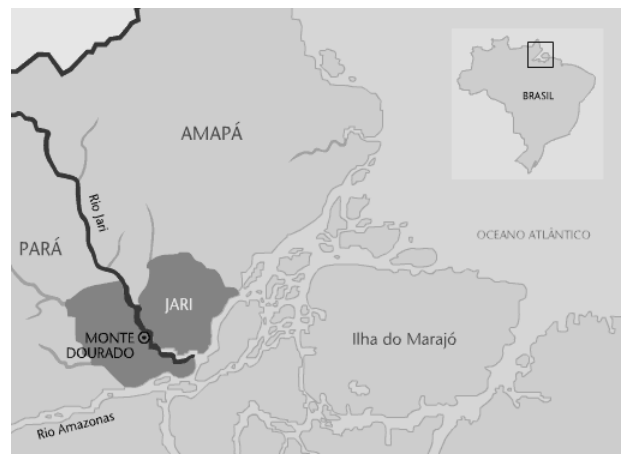

Figura 1. Localização geográfica da Floresta da Jari.

Figure 1. Geographical location of Jari Forest.

\section{Inventário pré-exploratório}

O inventário pré-exploratório foi realizado a uma intensidade de $100 \%$, considerando todas as árvores com DAP $\geq 50 \mathrm{~cm}$, na área experimental de 500 ha. No inventário, a floresta apresentou área basal de $13,56 \mathrm{~m}^{2} . \mathrm{ha}^{-1}$, sendo $8,56 \mathrm{~m}^{2} \cdot \mathrm{ha}^{-1}$ desse total de espécies de valor comercial. O volume total por hectare foi $173,18 \mathrm{~m}^{3}$, com 112,17 $\mathrm{m}^{3}$.ha ${ }^{-1}$ de espécies de valor comercial madeireiro. Foram encontradas 255 espécies, sendo 93 espécies comerciais para um total de 16.467 árvores, tendo em média 33 árvores por hectare (CARVALHO et al., 1987).

\section{Exploração florestal}

A exploração foi realizada em 400 ha. Foram exploradas árvores com DAP $\geq 60 \mathrm{~cm}$, de 42 espécies de valor comercial na região. Todas as informações relativas a esse subitem foram retiradas de Carvalho et al. (1987).

A exploração foi feita em três intensidades diferentes. A menor intensidade foi de $15 \%$ do volume total por hectare $\left(173,18 \mathrm{~m}^{3}\right)$ obtido no inventário pré-exploratório, correspondendo a $26 \mathrm{~m}^{3} \cdot \mathrm{ha}^{-1}$; a intensidade média foi de $25 \%$ do volume, correspondendo a $43 \mathrm{~m}^{3}$.ha-1; e a maior foi de $35 \%$ do volume, correspondendo a $61 \mathrm{~m}^{3} \cdot \mathrm{ha}^{-1}$. Em média foram exploradas três árvores por hectare na primeira intensidade, cinco na segunda e sete na terceira.

\section{Tratamento silvicultural}

Os tratamentos silviculturais foram realizados em 1994, nove anos após a exploração. O desbaste objetivou reduzir a competição entre árvores por espaço, luz e nutrientes, proporcionando aumento da sobrevivência, do crescimento e estabelecimento da regeneração natural das espécies de valor econômico.

As técnicas utilizadas foram o desbaste sistemático, que consistiu em eliminar árvores de espécies não comerciais a partir de um diâmetro mínimo de $15 \mathrm{~cm}$, até atingir a redução de área basal planejada, e o desbaste seletivo, que se resumiu na eliminação de árvores de espécies não comerciais 
cujas copas estivessem competindo com árvores de espécies comerciais selecionadas para futura extração. A determinação das áreas basais reduzidas foi feita com base nas tabelas de distribuição de área basal por espécie antes da exploração. Nessa redução da área basal foi considerada aquela inicialmente provocada pela exploração florestal, ou seja, o impacto ocasionado pela exploração foi incluído (COSTA et al., 2001).

A determinação do diâmetro mínimo das árvores a serem eliminadas em cada tratamento foi realizada da seguinte maneira: somou-se a área basal da maior para a menor classe de diâmetro até se obter um valor aproximado ao da área basal a ser reduzida. Tomou-se o cuidado, durante o planejamento, de evitar a eliminação ou o risco de extinção de qualquer espécie, preservando com isso a riqueza florística da comunidade. Cuidados especiais foram tomados para evitar o anelamento de árvores das espécies consideradas importantes para a fauna e para as atividades extrativistas.

A desvitalização foi realizada através da anelagem com entalhes, que consiste em um anel feito com golpes contínuos de machadinha em torno do fuste das árvores, sem a retirada da casca e com aplicação de Tordon 2,4 D, diluído em água a uma concentração de 10\%, pulverizado nos anéis (COSTA et al., 2001).

\section{Delineamento experimental}

Em 400 ha foram plotados três blocos de 48 ha, que compõem o experimento, totalizando uma área amostral de 144 ha. Todos os blocos têm bordaduras e foram deixadas faixas entre um bloco e outro e entre os blocos e as estradas permanentes. Os 100 ha restantes foram mantidos como testemunha.

O delineamento foi estruturado em blocos ao acaso com três repetições. As alternativas de manejo testadas foram combinações de intensidades de exploração com intensidade de desbastes. As intensidades de exploração foram representadas por porcentagens do volume extraído em relação ao volume total da floresta a partir do DAP de $60 \mathrm{~cm}$. Aplicaram-se intensidades de 15\% (intensidade leve), 25\% (intensidade média) e 35\% (intensidade pesada). Elas foram combinadas com dois tipos de intensidades de desbastes, o desbaste sistemático nas intensidades de $30 \%$ e $50 \%$ de redução da área basal original e o desbaste seletivo, ou de liberação de copas. Os tratamentos resultantes foram os seguintes:

- $\mathrm{T}_{1}$ - exploração de $15 \%$ do volume total das árvores de DAP $>60 \mathrm{~cm}$, sem redução da área basal após a exploração;

- $\mathrm{T}_{2}$ - exploração de $15 \%$ do volume total das árvores de DAP >60 cm, com redução de $30 \%$ da área basal original através de tratos silviculturais;

- $\mathrm{T}_{3}$ - exploração de $15 \%$ do volume total das árvores de DAP $>60 \mathrm{~cm}$, com redução de $50 \%$ da área basal original através de tratos silviculturais;

- $\mathrm{T}_{4}$ - exploração de $15 \%$ do volume total das árvores de DAP $>60 \mathrm{~cm}$, com redução de $70 \%$ da área basal original através de tratos silviculturais;

- $\mathrm{T}_{5}$ - exploração de $25 \%$ do volume total das árvores de DAP $>60 \mathrm{~cm}$, sem redução da área basal após a exploração;

- $\mathrm{T}_{6}$ - exploração de $25 \%$ do volume total das árvores de DAP $>60 \mathrm{~cm}$, com redução de $30 \%$ da área basal original através de tratos silviculturais;

- $\mathrm{T}_{7}$ - exploração de $25 \%$ do volume total das árvores de DAP $>60 \mathrm{~cm}$, com redução de $50 \%$ da área basal original através de tratos silviculturais;

- $\mathrm{T}_{8}$ - exploração de $25 \%$ do volume total das árvores de DAP $>60 \mathrm{~cm}$, com redução de $70 \%$ da área basal original através de tratos silviculturais;

- $\mathrm{T}_{9}$ - exploração de $35 \%$ do volume total das árvores de DAP $>60 \mathrm{~cm}$, sem redução da área basal após a exploração;

- $\mathrm{T}_{10}$ - exploração de $35 \%$ do volume total das árvores de DAP >60 cm, com redução de $30 \%$ da área basal original através de tratos silviculturais.

- $\mathrm{T}_{11}$ - exploração de $35 \%$ do volume total das árvores de DAP $>60 \mathrm{~cm}$, com redução de $50 \%$ da área basal original através de tratos silviculturais;

- $\mathrm{T}_{12}$ - exploração de $35 \%$ do volume total das árvores de DAP $>60 \mathrm{~cm}$, com redução de $70 \%$ da área basal original através de tratos silviculturais.

Dentro dos blocos experimentais, a exploração foi feita em faixas, sendo cada faixa (200 m x $800 \mathrm{~m}$ ) com uma intensidade diferente. Fora dos blocos, a intensidade de exploração foi de $35 \%$ do volume (Figura 2). 


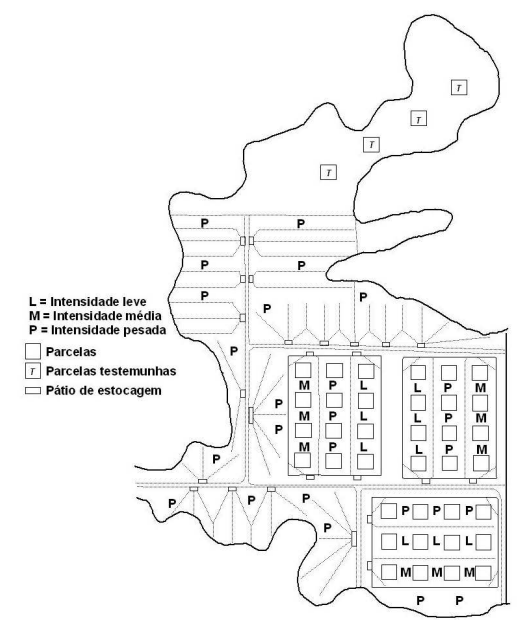

Figura 2. Croqui do experimento (L: intensidade leve; M: intensidade média; P: intensidade pesada). Figure 2. Experimental design (L: light intensity; M: medium intensity; P: heavy intensity).

\section{Monitoramento da floresta}

$\mathrm{Na}$ área experimental de 500 hectares foram locadas 40 parcelas permanentes de um hectare cada, sendo trinta e seis na área explorada e quatro na área não explorada. A primeira medição foi realizada em 1984, a exploração em 1985 e as remedições em 1986, 1988, 1990, 1994, 1996 e 2004.

A metodologia empregada para instalação e coleta de dados das unidades amostrais (parcelas permanentes) foi desenvolvida e disponibilizada no Protocolo de Inventário para Monitorar o Crescimento e Produção de Florestas Tropicais, assim como também a criação de banco de dados utilizando-se o SFC (Sistema de Inventário Florestal Contínuo) (SILVA; LOPES, 1984).

\section{Procedimentos de medição}

Todas as árvores com diâmetro maior ou igual a $20 \mathrm{~cm}$ foram medidas periodicamente nas parcelas. As parcelas permanentes possuem forma quadrada, com dimensões de $100 \mathrm{~m}$ x $100 \mathrm{~m}$ (1 ha), e foram internamente subdivididas em 100 unidades de observações de $10 \mathrm{~m}$ x $10 \mathrm{~m}$ (subparcelas), para facilitar a localização e o controle de cada árvore a ser medida e monitorada. As subparcelas foram numeradas de 1 a 100, iniciando sempre pelo canto sudoeste. As variáveis tomadas para cada árvore, segundo Silva e Lopes (1984), são: número da árvore, classe de identificação do fuste (CIF), medição do diâmetro, identificação botânica, forma e iluminação da copa, presença e efeito dos cipós, situação silvicultural e coordenadas cartesianas.

\section{RESULTADOS E DISCUSSÃO}

Pode-se observar que o número de árvores $(\mathrm{N})$, a área basal $(\mathrm{G})$ e o volume (V) das árvores aneladas variaram consideravelmente entre os tratamentos (Tabela 1). Na aplicação do tratamento foram aneladas em média 18,3 árvores.ha ${ }^{-1}, 2,1 \mathrm{~m}^{2} \cdot \mathrm{ha}^{-1}$ de área basal e $25,1 \mathrm{~m}^{3} \cdot \mathrm{ha}^{-1}$ de volume.

Foram anelados 495 indivíduos, pertencentes a 93 espécies. Destas, Geissospermum sericeum quinarana (81), Cecropia sciadophylla - torém (33), Mouriri callocarpa - muiraúba-amarela (31), Maytenus floribunda - xixuá-vermelho (29), Inga alba - ingá-xixi (20), não identificadas (18), Drypetes variabilis - Maparana (17), Inga spp. - Mimosaceae/ingá (16), Inga paraensis - Ingá-vermelho (15), Anaxagorea dolichocarpa - envira-branca (12), Pouteria spp. - Sapotaceae/pouteria (10) e Rinorea guianensis - acariquarana (10) representaram 59\% do total de indivíduos anelados.

As famílias com maior número de indivíduos anelados foram as seguintes: Apocynaceae, Mimosaceae, Moraceae, Melastomataceae, Celastraceae, Sapotaceae, Euphorbiaceae, Elaeocarpaceae, Annonaceae, Violaceae, Caesalpiniaceae, Lecythidaceae e Monimiaceae, correspondendo a $83 \%$ do número total de árvores aneladas.

Observa-se na tabela 2 que a quantidade de árvores mortas em consequência do anelamento 
variou consideravelmente entre os tratamentos. Dez anos após o anelamento, as parcelas dos tratamentos $\mathrm{T}_{8}, \mathrm{~T}_{3}, \mathrm{~T}_{11}$ e $\mathrm{T}_{4}$ foram as que apresentaram a maior efetividade, com a mortalidade variando de $90,3 \%$ a $84 \%$ das árvores aneladas. $\mathrm{O} \mathrm{T}_{7}$ foi o que mostrou a mais baixa efetividade, com cerca de $50 \%$.

Tabela 1. Características dos tratamentos silviculturais realizados em cada tratamento na área experimental da Floresta da Jari.

Table 1. Characteristics of silvicultural treatments carried out in each treatment at the experimental Forest of Jari.

\begin{tabular}{|c|c|c|c|c|c|c|c|c|c|c|c|c|c|c|}
\hline \multirow{2}{*}{ Área } & \multirow{2}{*}{ Variável } & \multicolumn{12}{|c|}{ Tratamentos } & \multirow{2}{*}{ Média } \\
\hline & & T1 & $\mathbf{T 2}$ & T3 & T4 & T5 & T6 & T7 & T8 & T9 & T10 & T11 & T12 & \\
\hline \multirow{4}{*}{$\begin{array}{l}\text { Total } \\
\text { (3 ha) }\end{array}$} & $\mathrm{N}$ & - & 71,00 & 70,00 & 25,00 & - & 59,00 & 38,00 & 72,00 & - & 91,00 & 33,00 & 36,00 & 55,00 \\
\hline & $\mathrm{G}\left(\mathrm{m}^{2}\right)$ & - & 8,96 & 9,39 & 3,37 & - & 5,97 & 5,59 & 8,11 & - & 8,41 & 3,70 & 3,90 & 6,38 \\
\hline & $\mathrm{V}\left(\mathrm{m}^{3}\right)$ & - & 108,19 & 113,65 & 39,48 & - & 68,21 & 67,55 & 96,56 & - & 97,01 & 43,05 & 44,57 & 75,36 \\
\hline & $\mathrm{DAP}(\mathrm{cm})$ & - & 36,32 & 37,62 & 40,04 & - & 33,71 & 41,11 & 33,90 & - & 31,08 & 34,65 & 35,66 & 36,01 \\
\hline \multirow{4}{*}{$\begin{array}{l}\text { Parcela } \\
\text { (ha) }\end{array}$} & $\mathrm{N}$ & - & 23,67 & 23,33 & 8,33 & - & 19,67 & 12,67 & 24,00 & - & 30,33 & 11,00 & 12,00 & 18,33 \\
\hline & $\mathrm{G}\left(\mathrm{m}^{2}\right)$ & - & 2,99 & 3,13 & 1,12 & - & 1,99 & 1,86 & 2,70 & - & 2,80 & 1,23 & 1,30 & 2,13 \\
\hline & $\mathrm{V}\left(\mathrm{m}^{3}\right)$ & - & 36,06 & 37,88 & 13,16 & - & 22,74 & 22,52 & 32,19 & - & 32,34 & 14,35 & 14,86 & 25,12 \\
\hline & $\mathrm{DAP}(\mathrm{cm})$ & - & 36,11 & 38,28 & 44,35 & - & 33,79 & 40,85 & 34,31 & - & 36,56 & 39,75 & 44,21 & 38,69 \\
\hline
\end{tabular}

Isto se deve, em parte, às características morfológicas e dendrológicas das espécies Geissospermum sericeum (quinarana) e Mouriri callocarpa (muiraúba-amarela). Do total de 81 árvores aneladas de quinarana, 65 permaneceram vivas, o que representa uma mortalidade de apenas $20 \%$. Já árvores aneladas de muiraúba-amarela apresentaram mortalidade de $45 \%$. Geissospermum sericeum, da família Apocynaceae, possui o fuste bastante sulcado, o que dificultou a execução do anelamento e a aplicação do arboricida. No $\mathrm{T}_{7}$, das 38 árvores aneladas, 15 são quinarana e se mantêm vivas até o momento.

Tabela 2. Efetividade do anelamento com aplicação de arboricida, por tratamento (3 ha), na área experimental da Floresta da Jari.

Table 2. Effectiveness of girdling with application of arboricida per treatment ( $3 \mathrm{ha}$ ) in Jari Forest.

\begin{tabular}{|c|c|c|c|c|c|c|c|c|c|c|c|c|c|c|c|c|c|c|}
\hline \multirow{2}{*}{ Variável } & \multicolumn{18}{|c|}{ Tratamentos } \\
\hline & \multicolumn{2}{|l|}{2} & \multirow[t]{2}{*}{3} & \multicolumn{3}{|c|}{4} & \multicolumn{2}{|l|}{6} & \multicolumn{2}{|l|}{7} & \multicolumn{2}{|l|}{8} & \multicolumn{2}{|l|}{10} & \multirow[t]{2}{*}{11} & \multicolumn{3}{|c|}{12} \\
\hline \multicolumn{17}{|c|}{ Árvores mortas dois anos após a aplicação dos tratamentos - 1996} & & \\
\hline $\mathrm{N}$ & 51,0 & 71,8 & 46,0 & 65,7 & 21,0 & 84,0 & 47,0 & 79,7 & 15,0 & 39,5 & 62,0 & 86,1 & 65,0 & 71,4 & 26,0 & 78,8 & 22,0 & 61,1 \\
\hline $\mathrm{G}\left(\mathrm{m}^{2}\right)$ & 5,5 & 61,4 & 4,3 & 45,5 & 2,9 & 84,8 & 4,6 & 77,7 & 2,3 & 41,8 & 6,5 & 79,6 & 4,8 & 56,6 & 3,1 & 82,6 & 2,2 & 57,0 \\
\hline $\mathrm{V}\left(\mathrm{m}^{3}\right)$ & 65,4 & 60,4 & 48,9 & 43,1 & 33,6 & 85,1 & 52,6 & 77,2 & 28,5 & 42,2 & 76,1 & 78,8 & 52,1 & 36,2 & 84,2 & 25,2 & & 56,5 \\
\hline $\mathrm{D}(\mathrm{cm})$ & 35,6 & & 30,8 & & 38,0 & & 32,8 & & 42,3 & & 34,3 & & 31,1 & 36,4 & & 32,8 & & \\
\hline \multicolumn{19}{|c|}{ Árvores mortas dez anos após a aplicação dos tratamentos - 2004} \\
\hline $\mathrm{N}$ & 56,0 & 78,9 & 60,0 & 85,7 & 21,0 & 84,0 & 49,0 & 83,1 & 19,0 & 50,0 & 65,0 & 90,3 & 72,0 & 79,1 & 28,0 & 84,8 & 26,0 & 72,2 \\
\hline $\mathrm{G}\left(\mathrm{m}^{2}\right)$ & 6,6 & 73,9 & 7,5 & 80,3 & 2,9 & 84,8 & 4,7 & 79,4 & 3,1 & 56,1 & 6,7 & 82,8 & 5,4 & 64,0 & 3,2 & 86,1 & 2,6 & 67,3 \\
\hline $\mathrm{V}\left(\mathrm{m}^{3}\right)$ & 79,7 & 73,7 & 91,0 & 80,1 & 33,6 & 85,1 & 53,6 & 78,6 & 38,9 & 57,5 & 78,8 & 81,6 & 59,1 & 60,9 & 37,5 & 87,1 & 29,5 & 66,1 \\
\hline $\mathrm{D}(\mathrm{cm})$ & 42,4 & & 38,4 & & 38,0 & & 29,1 & & 44,0 & & 33,1 & & 30,0 & & 32,0 & & 35,4 & \\
\hline \multicolumn{19}{|c|}{ Árvores vivas dez anos após a aplicação dos tratamentos - 2004} \\
\hline $\mathrm{N}$ & 14,0 & 19,7 & 10,0 & 14,3 & 4,0 & 16,0 & 10,0 & 16,9 & 18,0 & 47,4 & 8,0 & 11,1 & 17,0 & 18,7 & 6,0 & 18,2 & 10,0 & 27,8 \\
\hline $\mathrm{G}\left(\mathrm{m}^{2}\right)$ & 2,2 & 24,3 & 2,0 & 21,3 & 0,6 & 16,9 & 1,3 & 21,8 & 2,6 & 45,8 & 1,5 & 18,0 & 3,0 & 35,2 & 0,6 & 16,2 & 1,4 & 35,6 \\
\hline $\mathrm{V}\left(\mathrm{m}^{3}\right)$ & 26,4 & 24,4 & 24,6 & 21,7 & 6,8 & 17,3 & 15,4 & 22,6 & 30,2 & 44,7 & 18,4 & 19,0 & 37,6 & 38,8 & 6,8 & 15,8 & 16,7 & 37,5 \\
\hline $\mathrm{D}(\mathrm{cm})$ & 40,6 & & 50,7 & & 39,4 & & 46,4 & & 45,8 & & 55,5 & & 59,3 & & 34,1 & & 43,5 & \\
\hline
\end{tabular}

Nota: Em cada tratamento, a coluna da esquerda corresponde ao valor absoluto e o da direita o valor relativo da variável considerada.

Esses resultados são esperados para árvores com fustes sulcados ou canelados e com exsudação. Resultado semelhante foi relatado por Jardim et al. (1996) e Oliveira (2005), que concluíram que o anelamento profundo com aplicação de óleo diesel tem efeito muito pequeno em árvores com essas características.

Oliveira et al. (2006) avaliaram durante 21 anos as mudanças na estrutura horizontal de uma área 
de 136 ha na Floresta Nacional do Tapajós provocadas pela exploração de madeira e tratamentos silviculturais, e concluíram que, quando os tratamentos silviculturais não foram aplicados, a floresta original apresentou muito baixa reconstituição quanto ao número de árvores e área basal. Segundo os autores, a aplicação dos tratamentos silviculturais reduziria a competição entre as árvores remanescentes após o fechamento do dossel, facilitando o ingresso de novas árvores na comunidade.

De maneira geral, o anelamento com a aplicação do arboricida mostrou maior efeito em função da espécie do que com o tamanho das árvores. Árvores com diâmetro inferior a $50 \mathrm{~cm}$ apresentaram mortalidade em torno de $61 \%$ a $90 \%$. Nas classes diamétricas superiores, apesar de o número de árvores aneladas ter sido bem menor, a taxa de mortalidade foi em torno de $69 \%$ a $100 \%$, a exceção para a classe de 90-100 cm de DAP, que, mesmo após dez anos da aplicação, apresentou 0\% de mortalidade (Figura 3). Entretanto, esta última informação se refere a um único indivíduo de quinarana.

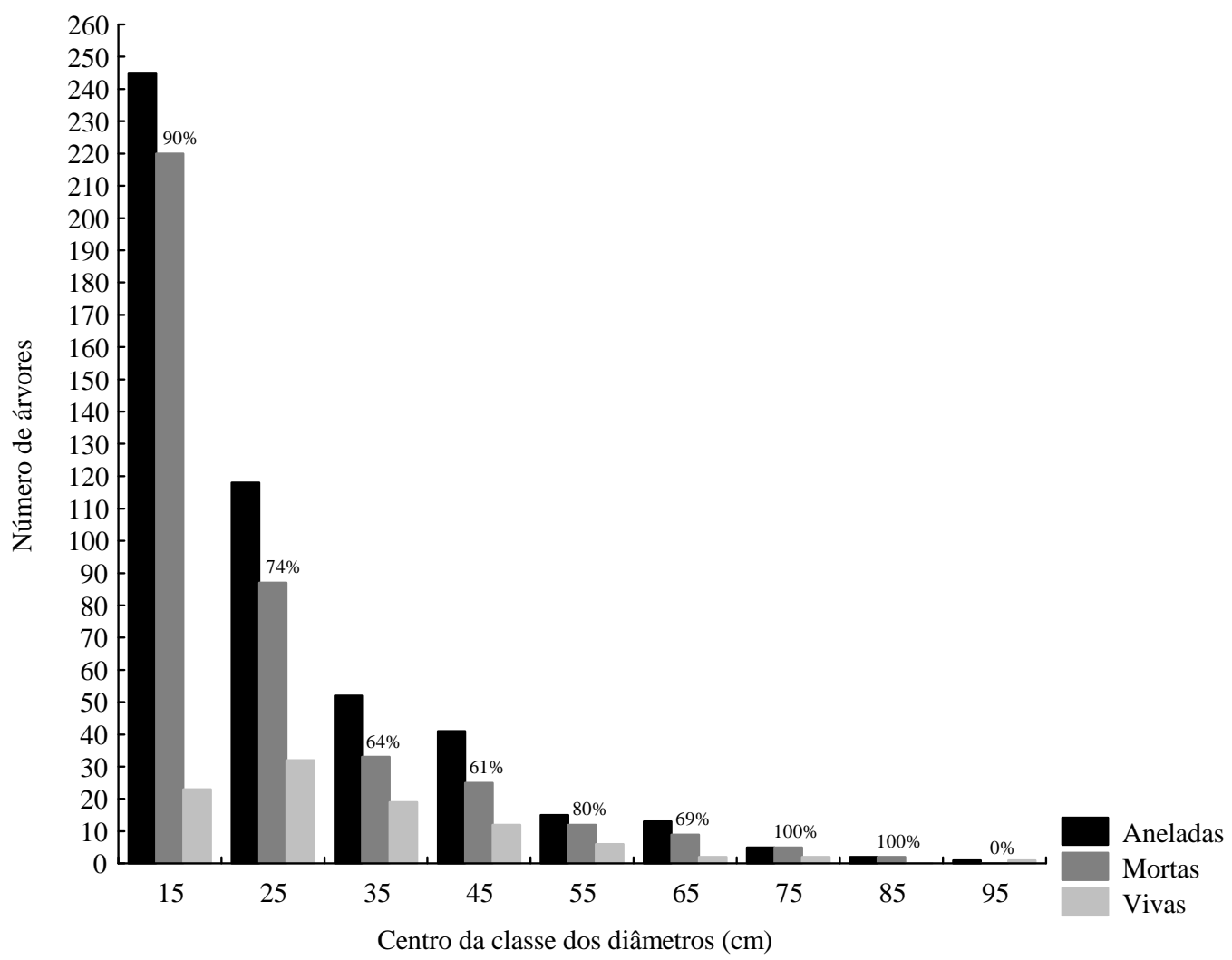

Figura 3. Número de árvores aneladas, desvitalizadas (mortas) e sobreviventes, por classe diamétrica, incluindo todos os tratamentos - 27 ha (Floresta da Jari).

Figure 3. Number of trees girdled, devitalized (dead) and survivors, by diameter class, including all treatments - 27 ha (Jari Forest).

Essas taxas de mortalidade contradizem os resultados obtidos por Oliveira (2005), em que o anelamento com a aplicação do arboricida mostrou maior efeito nas árvores com diâmetro inferior a $40 \mathrm{~cm}$, que apresentaram sobrevivência em torno de 14 a 29\%. Nas classes diamétricas superiores, a taxa média de sobrevivência ficou em torno de 44\%, com um máximo de 73\% na classe de 50-60 cm de DAP, nove anos após a aplicação do anelamento.

Os resultados obtidos neste trabalho também contrariam as afirmações de Dubois (1971) acerca da ineficiência do método para grandes árvores e são compatíveis aos encontrados por Jardim et al. (1996), que observaram, 3,5 anos após a aplicação dos tratamentos, que 74,1\% das árvores aneladas com DAP maior ou igual a $45 \mathrm{~cm}$ haviam morrido.

No estudo de Sandel e Carvalho (2000), o anelamento foi mais eficaz nas árvores com DAP 
entre $35 \mathrm{~cm}$ e $50 \mathrm{~cm}$, desde o segundo até o quinto ano após o tratamento. Não houve aplicação de arboricida. Neste trabalho, a taxa de mortalidade foi aumentando com o passar dos anos. No primeiro ano, a taxa de mortalidade foi de $25 \%$, no segundo foi de $50 \%$, no terceiro foi de $68,75 \%$, no quarto foi de $81,25 \%$ e no quinto ano foi de $85,42 \%$. Aos 17 anos após a aplicação do tratamento, a taxa de mortalidade chegou a atingir $95,8 \%$.

Pariona et al. (2001), estudando a efetividade de três tratamentos de anelamento na Bolívia, concluíram que o anelamento sem aplicação de arboricida causou a mortalidade de somente $10 \%$ das árvores tratadas, treze meses depois da aplicação. Os autores comentam que o principal motivo da baixa efetividade foi a cicatrização do anel em muitas das árvores tratadas sem arboricida, que permitiu a rápida reconexão dos tecidos cortados. Além disso, muitas árvores que não receberam arboricida produziram rebrotos no fuste, abaixo do anel. Ainda que esses rebrotos não tenham efeito sobre as árvores liberadas, com o tempo se converterão em fustes mal formados de espécies não comerciais, que continuarão ocupando espaço e reduzindo a qualidade do povoamento.

\section{CONCLUSÕES}

- $\quad \mathrm{O}$ anelamento com a aplicação do arboricida foi mais eficiente em função da espécie do que o tamanho das árvores, e para as classes diamétricas superiores.

\section{REFERÊNCIAS}

CARVAlHO, J. O. P.; LOPES, J. C. A.; SILVA, J. N. M.; COSTA, H. B.; MALCHER, L. B.; CARVALHO, M. S. P. Pesquisas com vistas ao manejo de matas nativas na região do rio Jari. Belém, PA. Embrapa - CPATU, 1987. 21 p.

COSTA, D. H. M.; SILVA, S. M. A. da; SILVA, J. N. M. Efetividade e custo do desbaste com aplicação de arboricida em floresta natural na região do Tapajós, Pará, e Jari, Amapá. In: Silva, J. N. M.; Carvalho, J. O. P. de; Yared, J. A. G. (eds.). A silvicultura na Amazônia Oriental: contribuições do projeto Embrapa/DFID. Belém: Embrapa Amazônia Oriental/DFID, 2001, p. 339 - 352.

DE GRAAF, N. R.; POELS, R. L. H.; Van ROMPAEY, R. S. A. R. Effect of silvicultural treatments on growth and mortality of rainforest in Suriname over long periods. Forest Ecology and Management, Amsterdam, v.124, p. 123 - 135, 1999.

DUBOIS, J. L. C. Silvicultural research in the Amazon. Rome: FAO, 1971, 53p. (Technical Report, 3).

JARDIM, F. C. S.; SOUZA, A. L.; BARROS, N. F.; MACHADO, C. C.; SILVA, E.; SILVA, A. F. Técnica de abertura do dossel por anelamento de árvores na Estação Experimental de Silvicultura do INPA, Manaus - AM. B. FCAP, Belém, 1996, v. 25, p. 91 - 104.

OLIVEIRA, L. C. Efeito da exploração madeireira e de diferentes intensidades de desbaste sobre a dinâmica da vegetação de uma área de 136 ha na Floresta Nacional do Tapajós. 2005. 195 f. Tese (Doutorado em Ciências Florestais) - Escola Superior de Agricultura “Luiz de Queiroz", Piracicaba, 2005.

OliveIRA, L. C. de; COUTO, H. T. Z do; SILVA, J. N. M.; CARVALHO, J. O. P. de. Efeito da exploração de madeira e tratamentos silviculturais sobre a estrutura horizontal de uma área de 136 ha na Floresta Nacional do Tapajós, Belterra-Pará. Revista de Ciências Agrárias, n. 46, p. 195 - 214, 2006.

PARIONA, W.; FREDERICKSEN, T.; LICONA, J. C. Comparación de tres tratamientos para el mejoramiento de rodales en dos tipos de bosque bolivianos. Bolivia: Proyecto BOLFOR, 2001, 16 p. (Documento Técnico, 102).

SANDEL, M. P.; CARVALHO, J. O. P. Anelagem de árvores como tratamento silvicultural em floresta naturais da Amazônia brasileira. Revista Ciências Agrárias, n. 33, p. 9 - 32, 2000.

SILVA, E. J. V. Dinâmica de florestas manejadas e sob exploração convencional na Amazônia oriental. 2004. 171 f. Tese (Doutorado em Ciências da Engenharia Ambiental) - Universidade de São Paulo, São Carlos, 2004. 
SILVA, J. N. M.; LOPES, J. C. A. 1984. Inventário florestal contínuo em florestas tropicais: a metodologia utilizada pela EMBRAPA-CPATU na Amazônia brasileira. Belém: Embrapa-CPATU, 1984, 36 p. (Embrapa-CPATU. Documentos, 33).

SILVA, S. M. A. da; SILVA, J. N. M.; BAIMA, A. M. V.; LOBATO, N. M.; THOMPSON, I. S.; COSTA-FILHO, P. P. Impacto da exploração madeireira em floresta de terra firme no município de Moju, estado do Pará. In: Silva, J. N. M.; Carvalho, J. O. P. de; Yared, J. A. G. A silvicultura na Amazônia Oriental: contribuições do projeto Embrapa/DFID. Belém: Embrapa Amazônia Oriental/DFID, 2001, p. 309 - 323. 
